\title{
Facility Readiness for Childhood Routine Immunization Services in Primary Healthcare Centres in Benin City, Edo State, Nigeria
}

\author{
Uwaibi E. Noel \\ Department of Community Health and Community medicine \\ Edo University Iyamho, km7 Auchi-Abuja express road, Auchi, Edo state \\ Nigeria
}

\begin{abstract}
Background and objective of study: immunization remains an essential strategy for reducing mortalities and morbidities due to vaccine preventable diseases. Immunization service delivery is dependent on planning of immunization sessions, and Supervision of the immunization process as this is very important in order to achieve immunization coverage target.

Materials and Methods: A descriptive cross sectional study was utilised. This was done using an observational check list guide which was developed by the researcher and utilised for assessment of facility readiness for routine immunization service delivery. It was used to assess the facilities, amenities and logistics utilised in the immunization services process and also the functionality of the amenities. It was utilised in 35 Primary health care centres in the 3 Local Government Areas.
\end{abstract}

Results: Thirty two (91.4\%) had sufficient staff offering immunization and 22 (62.9\%) had sufficient seats for clients. All the facilities had supervisory visits by the government. Thirty three (94.3\%) had health talks before immunization and 25 (71.4\%) had an alternate functional source of power supply readily available

Conclusion: Only 3 of the PHCs were ready for routine immunization service delivery while 32 were partially ready for immunization.

Keywords: Facility Readiness, Vaccine Preventable Diseases, Cold Chain, Routine Immunization, Primary Healthcare Workers.

\section{INTRODUCTION}

Immunization is an essential strategy to improvement in child's wellbeing. Providing vaccine as a means to achieving immunization coverage targets without giving adequate attention to quality and utilization of immunization services does not guarantee a reduction in childhood disease morbidity and mortality due to VPDs in children. Routine immunization has critical component such as immunization policy, standards and guidelines, governance, organization and management, human resources, vaccine, cold chain and logistics management, service delivery, communication and community partnership, data generation/use and sustainable financing.[1] Immunization service delivery is also dependent on planning of immunization sessions, and Supervision of the immunization process[2] Other factors are location of the primary health facilities, quality of care available, attitude of mothers/caregivers, population of health workers, accessibility to vaccination centres, and availability of safe needles and syringes[3]Finding from an Ethiopian study revealed that poor infrastructure of the health facilities was the main reasons behind the country's low immunization coverage.[4]The growing complexity of immunization programs increases the need for well-trained, capable health workforce, with requisite technical skills to administer an increasing number of vaccines to more 
children and expanded age groups. This will lead to management and supervisory skills becoming increasingly important as vaccine costs rise and data management responsibilities increases. [1]

In many parts of Nigeria, there is shortage of qualified personnel in PHC facilities. This has resulted in unavailability of 24 hour services in many PHC facilities. Poor utilization of PHC facilities in favour of unskilled health practitioners has largely contributed to the poor health indices in the country. Utilization of PHC services is directly affected by the readiness of PHC facilities to deliver health services in terms of adequate staffing and equipment. This low level of facility readiness in PHCs has contributed to poor utilization of PHC services by mothers. [1]

Research has also shown inadequacies with the health care facility readiness for implementation of routine immunization, as $35.7 \%$ of the PHCs suffer shortage of one or more vaccines, and majority of the health facilities have no supervision.[2]These could result in fragile primary health care system, suboptimal service delivery at health facilities, gaps in health workers skills as well as weaknesses in the RI program.[5] In Nigeria, the weaknesses in the RI system are mainly structural and logistical, with most of the states having homogeneity in identified barriers. Inadequate transportation, poor cold chain, families and financial barriers are the leading issues across all states. Other challenges include lack of accountability. Limited political will benefit for RI support and problems of fund disbursement, over decentralization of designated funds across states and LGAs. Overarching issues of structure and governance of the health facilities also affect supply and service delivery at every level. [6] This could lead to vaccine stock outs in health facilities and increased missed opportunity for childhood immunization and resultant increases in illness in children due to VPDs.

Successful cold chain and logistics management requires attention to many considerations, such as adequate fuel for vehicles and transportation like bicycles with cold chain boxes /vaccine carriers to ensure continuous running of cold chain system. Fuel and maintenance costs are often underestimated, and decisions to fund them are usually made by the local governments, which may have other priorities. Resources allocated for the RI logistics are most times diverted for other uses. In addition, maintaining vaccines at proper temperatures has become more complex than in the past as some new vaccines are inactivated by exposure to freezing while other vaccines (those that have been in use for decades) are damaged by heat exposure. With the unit cost of newer vaccines far more expensive than those of the original complement of EPI vaccines, poor vaccine-handling practices would have large financial consequences [1] these circumstances may lead to unreliable delivery of supplies and vaccine stock-outs, poor health indices and limited achievement of the Sustainable Development Goals (SDGs).

Expanded Programme on Immunization (EPI) evaluations has focused more on population coverage, and few studies have assessed facility and routine immunization at the point of service provision. [7]It is expected that findings from the study will help encourage research and provide information on facility readiness for routine immunization in primary health care centre. The study will provide a baseline for tracking progress in subsequent implementation of immunization services over time. Effective monitoring and evaluation of childhood immunization programmes are necessary as in their absence, vaccination rates may dwindle unnoticed for some time before increased incidence of target diseases are observed.

\section{MATERIALS AND METHODS}

2.1 study area: The study was carried out in Primary Health Care facilities across the three Local Government Areas in Benin City, Edo State, Nigeria. Benin City comprises three Local Government Areas, namely, Oredo, Egor, and Ikpoba Okha. Oredo, Egor, and Ikpoba Okha LGAs have 12, 10 and 10 political wards respectively. There are 35 Primary Health Care facilities in Benin City with 10, 7, and 18 PHCs in Oredo, Egor and Ikpoba Okha LGAs, respectively, and Routine Immunization (RI) activities are carried out in these PHCs.

2.2 study design: This was a cross-sectional descriptive (qualitative) study design, Facility readiness for RI was assessed in the Primary Health Care Centres (PHC), using an observational checklist parameter such as logistics, cold chain equipment, transportation, immunization sessions, supervision of the immunization sessions was assessed for this study.

Sample size was determined using the formula for studying single proportion. [8]. Based on documentation of previous study conducted in 2013 in Jamnagar district, India [2] a proportion of 98.0\%, was used as proportion of PHCs in which immunization sessions where held as planned in the previous months. A precision of 5\% was used and correction of non- response rate was made thus a total of 35 PHC was utilised for the study

\subsection{Observations}

This was carried out using the observational check list guide which was developed by the researcher and utilised for assessment of facility readiness for routine immunization service delivery. It was used to assess the facilities, amenities and logistics utilised in the immunization services process and also the functionality of the amenities. It was utilised in 35 PHCs in the 3 LGAs. It 
involved observing the health centres for facilities required for immunization such as the freezers, thermometers, vaccine vial monitors, checking their registers for properly filled forms and charts and also observing the health workers as they carried out the immunization process for injection safety practices.

The questionnaires were screened for completeness by the researcher, coded and entered into the IBM SPSS statistics 20.0 software. The categorical variables (sex, educational status, ethnicity, marital status, etc) were presented as frequencies and percentages while numerical variables (age, and years in service) that were normal in distribution were expressed as mean (standard deviation). Statistical tests such as independent were carried out to compare difference in means of numerical data (such as age in years of the respondents). The Chi-squared test of association was used to test statistical association between sociodemographic variables of the respondents and the knowledge of immunization, knowledge and practice of injection safety. The Fisher's exact test was used in instances where the total expected cell frequencies less than five is more than $20 \%$. The binary logistic regression was modelled to explore and identify significant predictors of quality of routine immunization services at the Primary Health Facilities in the LGAs .The level of significance was set at $p<0.05$.

\subsection{Scoring for facility readiness for routine immunization delivery in PHCs}

Assessment of facility structural inputs was done with an observational checklist based on the availability and correctly filled registers, equipment and infrastructure in the various primary health care facilities. For each item a score of ' 2 ' was given if available and functional, 1 if available but not functional, and score of 0 if not available. The scores were summed up giving a maximum possible score of 74 and minimum of 0 . The total was converted to percentage and classified thus: Not ready: 0 $39.9 \%$, partially ready: $40.0-69.9 \%$ and Ready: $70.0-100 \%$.

\subsection{Ethical considerations}

Ethical clearance to conduct this research was sought and obtained from the University of Benin Teaching Hospital Ethics and Research Committee and Permission was sought from the Permanent Secretary, Edo State Hospital Management Board and Chairpersons of the Local Government Areas.

\subsection{RESULTS}

Thirty five PHCs were utilised for the study, the following domains were assessed: Management of cold chain system in the Health facilities, Logistics for routine immunization at the health facility and Injection safety and biomedical waste disposal at the health facility

Table 1: Management of cold chain system in the Health facilities

\begin{tabular}{|c|c|c|}
\hline Parameters & $\begin{array}{l}\text { Available } \\
\text { Freq }(\%)\end{array}$ & $\begin{array}{l}\text { Not available } \\
\text { Freq }(\%)\end{array}$ \\
\hline Cold chain boxes & $31(88.6)$ & $4(11.4)$ \\
\hline Cotton wool & $35(100.0)$ & $0(0.0)$ \\
\hline Vaccine carrier & $29(82.9)$ & $6(17.1)$ \\
\hline Functional refrigerator /freezer & $32(91.4)$ & $3(8.6)$ \\
\hline ILR/DF placed on wooden blocks & $25(71.4)$ & $10(28.6)$ \\
\hline ILR/DF $10 \mathrm{~cm}$ away from wall & $30(85.7)$ & $5(14.3)$ \\
\hline Connected to functional voltage stabilizer & $28(80.0)$ & $7(20.0)$ \\
\hline Functional thermometer inside ILR & $11(31.4)$ & $24(68.6)$ \\
\hline Functional thermometer inside DF & $4(11.4)$ & $31(88.6)$ \\
\hline$<5 \mathrm{~mm}$ frost inside ILR & $5(14.3)$ & $30(85.7)$ \\
\hline$<5 \mathrm{~mm}$ frost inside DF & $5(14.3)$ & $30(85.7)$ \\
\hline Record of Power failure & $32(91.4)$ & $3(8.6)$ \\
\hline Record of defrosting & $32(91.4)$ & $3(8.6)$ \\
\hline Presence of temperature log book & $30(85.7)$ & $5(14.3)$ \\
\hline All vaccine vials placed correctly arranged inside ILR & $35(100.0)$ & $0(0.0)$ \\
\hline
\end{tabular}


International Journal of Advances in Scientific Research and Engineering (ijasre), Vol 6 (4), April -2020

\begin{tabular}{lll}
\hline All vaccine vials placed inside labelled cartons & $31(88.6)$ & $4(11.4)$ \\
Food other than vaccines inside ILR 1 & $8(22.9)$ & $27(77.1)$ \\
Diluents placed inside ILR at least 24 hours before distribution & $23(65.7)$ & $12(34.3)$ \\
Correct placement of Ice packs inside DF & $28(80.0)$ & $7(20.0)$ \\
RI vaccine stored inside DF & $29(82.9)$ & $6(17.1)$ \\
Vaccine vial monitor & $35(100.0)$ & $0(0.0)$ \\
Temperature recorded regularly on local schedule & $24(68.6)$ & $11(31.4)$ \\
Signature of MO in temperature log book & $20(57.1)$ & $15(42.9)$ \\
ILR Temperature between +2 to $+8^{\prime} \mathrm{C}$ & $6(17.1)$ & $29(82.9)$ \\
DF Temperature -15 to -25'C & $4(11.4)$ & $31(88.6)$ \\
Registered - $2^{\circ}$ to- $8^{\circ} \mathrm{C}$ at all times previous month & $20(57.1)$ & $15(42.9)$ \\
Vaccine reconstituted just before immunization, & $32(91.4)$ & $3(8.6)$ \\
Time of reconstitution written on vial, & $20(57.1)$ & $15(42.9)$ \\
Reconstituted vaccine used within 4 hours & $33(94.3)$ & $2(5.7)$ \\
Appropriate use of $0.5 \mathrm{ml}$ and $0.1 \mathrm{ml}$ syringe & $35(100.0)$ & $0(0.0)$ \\
Correct selection of sites for vaccination & $33(94.3)$ & $2(5.7)$ \\
\hline
\end{tabular}

*DF-Deep freezer, ILR- ice lined refrigerator, MO- medical officer

Most, $31(88.6 \%)$ of the PHCs had cold chain boxes and all had cotton wool and sharps boxes. Twenty nine (82.9\%) had a functional vaccine carrier. Majority $25(71.4 \%)$ placed the refrigerator on wooden blocks and $30(85.7 \%)$ had it at least $10 \mathrm{~cm}$ away from the wall. Eleven (31.4\%) had a functional thermometer inside the ice lined refrigerator (ILR) while 4 (11.4\%) had one in the deep freezer (DF). All vaccines and vials were correctly arranged in the ILR in all the PHCs and vaccines were reconstituted and used within 4 hours in 33 (94.3\%) of the PHCs. All the facilities had vaccines with vaccine vial monitors.

Table 2: Logistics for routine immunization at the Health facility

\begin{tabular}{|c|c|c|}
\hline Logistics & $\begin{array}{l}\text { Yes } \\
\text { Freq }(\%)\end{array}$ & $\begin{array}{l}\text { No } \\
\text { Freq }(\%)\end{array}$ \\
\hline \multicolumn{3}{|l|}{ Structural inputs } \\
\hline Sufficient staff offering immunization & $32(91.4)$ & $3(8.6)$ \\
\hline Means of transportation to outreach activities & $35(100.0)$ & $0(0.0)$ \\
\hline Presence of posters in reception/nursing station & $30(85.7)$ & $5(14.3)$ \\
\hline Availability of map of catchment area & $19(54.3)$ & $16(45.7)$ \\
\hline Availability of Session plan & $18(51.4)$ & $17(48.6)$ \\
\hline Availability of Supervision plan & $15(42.9)$ & $20(57.1)$ \\
\hline Availability of Drop out chart & $12(34.3)$ & $23(65.7)$ \\
\hline Availability of RI plotted graphs (monthly for $2015 / 2016$ ) & $19(54.3)$ & $16(45.7)$ \\
\hline Available RI - Hmis forms & $19(54.3)$ & $16(45.7)$ \\
\hline Counterfoils from previous session available at session site; & $32(91.4)$ & $3(8.6)$ \\
\hline Immunization and $\mathrm{MCH}$ register available at session site, & $33(94.3)$ & $2(5.7)$ \\
\hline Filled Supervisory forms at PHCs & $20(57.1)$ & $15(42.9)$ \\
\hline Supervisory visit conducted by Govt. & $35(100.0)$ & $0(0.0)$ \\
\hline Presence of health officials in last calendar month & $22(62.9)$ & $13(37.1)$ \\
\hline
\end{tabular}


International Journal of Advances in Scientific Research and Engineering (ijasre), Vol 6 (4), April -2020

\begin{tabular}{lll}
\hline Supervisors make recommendation on improvement & $22(62.9)$ & $13(37.1)$ \\
$\begin{array}{l}\text { Supervisor reprimand for unsatisfactory work } \\
\text { Process inputs }\end{array}$ & $26(74.3)$ & $9(25.7)$ \\
Seats sufficient for clients & $22(62.9)$ & $13(37.1)$ \\
Toilets facilities available & $35(100.0)$ & $0(0.0)$ \\
Clients attended to on a first come first serve basis & $35(100.0)$ & $0(0.0)$ \\
Health talk before immunization & $33(94.3)$ & $2(5.7)$ \\
Availability of HCW & $35(100.0)$ & $0(0.0)$ \\
Open space/reception & $35(100.0)$ & $0(0.0)$ \\
Availability of blank immunization cards & $35(100.0)$ & $0(0.0)$ \\
Source of power supply generator or solar /electric power & $25(71.4)$ & $10(28.6)$ \\
\hline
\end{tabular}

Thirty two (91.4\%) had sufficient staff offering immunization and 22 (62.9\%) had sufficient seats for clients. All the facilities had supervisory visits by the government. Thirty three (94.3\%) had health talks before immunization and $25(71.4 \%)$ had an alternate functional source of power supply readily available.

Table 3: Injection safety and biomedical waste disposal at the health facility

\begin{tabular}{lll}
\hline Parameters & Available & Not available \\
& \multicolumn{1}{c}{$\mathbf{\text { Freq } \%}$} & Freq \% \\
\hline Injection safety & & \\
Recapping of needle & $28(80.0)$ & $7(20.0)$ \\
Presence of loose disposable needles and syringes & $17(48.6)$ & $18(51.4)$ \\
Evidence of attempt to sterilize disposable injection equipment & $0(0.0)$ & $35(100.0)$ \\
Presence of other infectious waste (other than used sharps) & $35(100.0)$ & $0(0.0)$ \\
Multi-dose vial with a needle left in the diaphragm & $14(40.0)$ & $21(60.0)$ \\
Overflowing or pierced sharps containers & $33(94.3)$ & $2(5.7)$ \\
Used sharps in an open container & $21(60.0)$ & $14(40.0)$ \\
Separate waste containers for sharps & $35(100.0)$ & $0(0.0)$ \\
Separate waste containers for infectious & $14(40.0)$ & $21(60.0)$ \\
Separate waste containers for non-infectious & $35(100.0)$ & $0(0.0)$ \\
Puncture resistant and leak proof sharps container & $35(100.0)$ & $0(0.0)$ \\
Puncture-resistant sharps container "in stock" & $35(100.0)$ & $0(0.0)$ \\
Running water and soap for washing hands & $24(68.6)$ & $11(31.4)$ \\
Alcohol-based hand rub for cleansing hands & $19(54.3)$ & $16(45.7)$ \\
Sharps containers awaiting final destruction completely closed & $9(25.7)$ & $26(74.3)$ \\
Full sharps containers stored safely away from public access & $11(31.4)$ & $24(68.6)$ \\
Bio Medical Wastes disposal & & \\
Disposal pit used only for disposal of sharp & $21(60.0)$ & $14(40.0)$ \\
Disposal of needle and syringe & $35(100.0)$ & $0(0.0)$ \\
Sharp boxes & $35(100.0)$ & $0(0.0)$ \\
\hline
\end{tabular}

Recapping of needles was done in $28(80.0 \%)$ of the facilities though all facilities disposed of used needles and syringes. 
Used needles and syringes were found littered on the floors and tables of the injection rooms (outside the safety box) in seven injection providing sites. Only $9(25.7 \%)$ of the facilities had sharp containers awaiting destruction completely closed and 11 $(31.4 \%)$ stored full sharp containers away from public access. Twenty one $(60.0 \%)$ had disposal pits for disposing sharps.

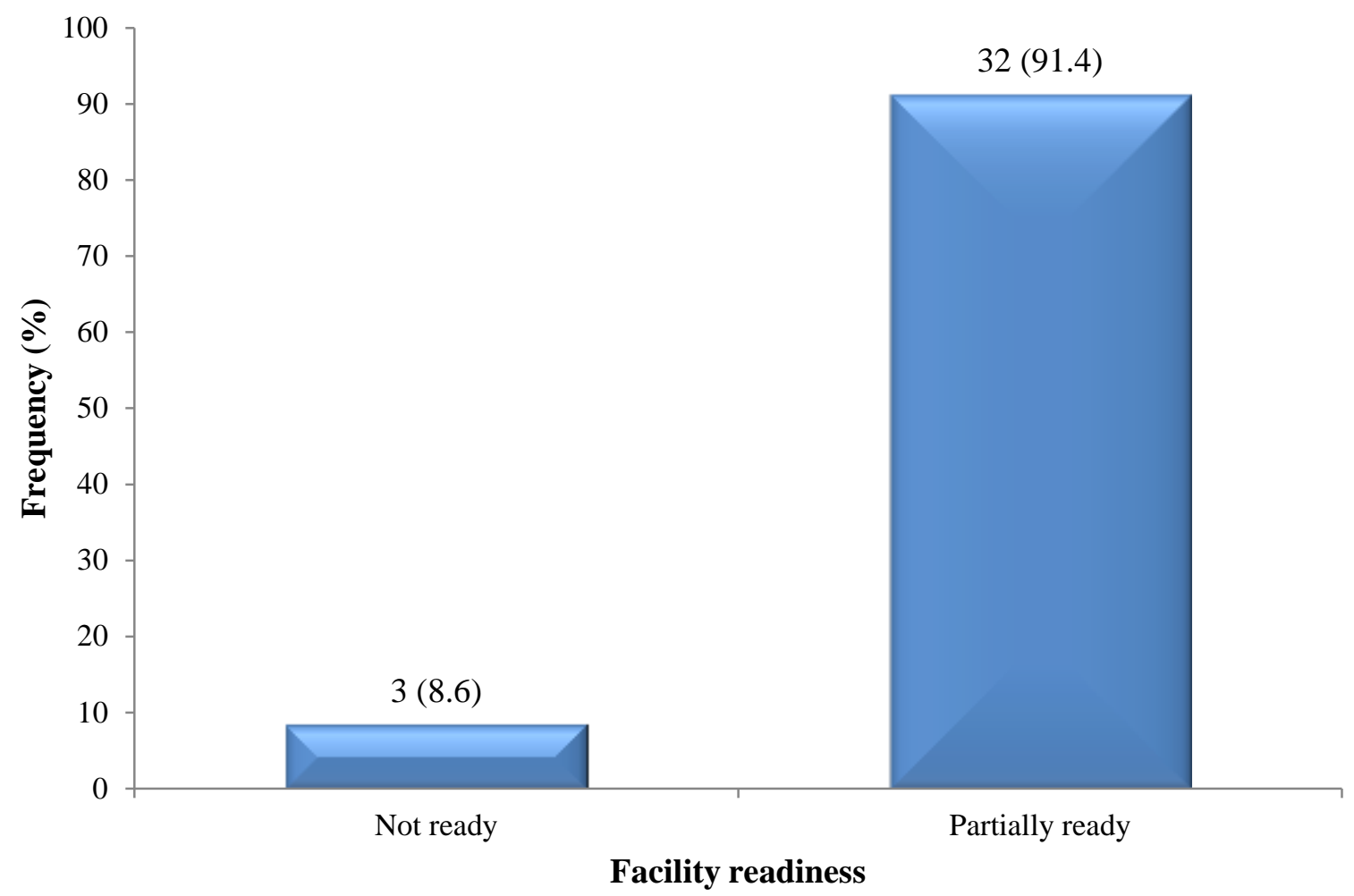

Figure 1: Composite score of facility readiness for childhood routine immunization

Only 3 of the PHCs were ready for routine immunization service delivery while 32 were partially ready for immunization.

\subsection{DISCUSSION}

Planning routine immunization entails a continuous process of analysing, evaluating progress, constraints and making decisions about reaching set program objectives. It was observed in this study that session plans for routine immunizations were available in only half of the PHCs studied. Furthermore maps of catchment areas, estimation of logistics, dropout chart which are valuable for improved planning of immunization services were not prepared in most of the PHCs. These findings are in contrast to those reported in Jamnagar district of India where all PHCs had planned immunization sessions. [2] Current findings are nonetheless improvements on those reported in another study carried out in India [9] where less than a quarter held immunization sessions. The implication of the extant finding is that poor planning of routine immunization sessions could lead to frequent vaccine stock outs, time wastage at the health centres and missed opportunity for vaccines

All health facilities were under the supervision of the government officials but less than half of the health facilities were not visited in the last month preceding the study. This finding is an improvement on the result gotten from a Cameroonian study where none of the health facilities studied had been visited in six months prior to the study.[7] Supervisors may prefer to visit only health facilities that are close to them, while health facilities in hard to reach areas remain unsupervised. This may partly explain the observation in this study. It may also be due to poor logistics, poor motivation of the supervisors and lack of expertise occasioned by limited funding.

Majority of the PHCs were at least partially ready for routine immunization services as noted in this study though all PHCs offered immunization services. This state of facility readiness may be due to continuous supervision and increased political will of the local government authorities and other stakeholder's commitment to the vaccination process. This finding may also be due to increase demand for routine immunization services by members of the community. Routine immunization is often available in 
most of the PHCs and it is affordable with less time spent in accessing PHC facilities than private hospitals and secondary health facilities. The partial readiness PHCs for immunization may be attributed to non-availability of equipment and facilities needed for immunization. Provision of the required equipment/facilities in the PHCs would enhance good performances by PHC workers which will in turn encourage utilization of PHC services, with a resultant reduction in morbidity and mortality in vaccine preventable disease in children coupled with improvement in child health indices.

Observations of this study revealed acute shortage of some cadres of health workers in most of the PHCs. The shortages may serve as impediment to achieving goals of achieving immunization coverage of $80 \%$ at ward/PHC level. Poor staff motivation was also mentioned as a factor influencing the implementation of the routine immunization. Being owed months of salaries leaves the health providers with no option other than seek ways and means to survive which could have deleterious effect on the implementation of routine immunization in PHCs.

This study revealed a system planned and earmarked to play a role in the attainment 'health for all' as declared at the Alma Ata, but burdened by weakness and challenges. There is need for identified lapses in the PHCs to be addresses if Sustainable Development Goal 3 (end preventable deaths of new-borns and under-5 children by 2030) is to be met.152-157Adoption and implementation of the 2013 Nigeria Routine Immunization Strategic Plan with an accountability framework offers an appropriate platform for reducing the inequalities in access, utilization and strengthening immunization services in the country. [10]

\section{Source of support: None \\ Conflict of interest: None}

\section{REFERENCES}

1. AK Shen, AR Fields, and MB McQuestion. The future of routine immunization in the developing world: challenges and opportunities. Global Health: Science and Practice. 2014; 2(4):381-92

2. M. Mithun. Assessment of routine immunization program at primary health centre level. National Journal of Medical Research. 2013; 3(4):319-23.

3. I. Abdulraheem, AT. Onajole, A. Jimoh and AR Oladipo. Reasons for incomplete vaccination and factors for missed opportunities among rural children. Journal of Public Health and Epidemiology. 2011;3 (4):194-203.

4. AA. Salah, GE. NegaBaraki, W Godana. Evaluation of the quality of Expanded Program on Immunization service delivery in Primary Health Care Institutions of Jigjiga Zone Somali Region, Eastern Ethiopia. European Journal of Preventive Medicine. 2015;3(4):117-123.

5. Paediatric Association of Nigeria Recommended routine immunization schedule for Nigerian children. Niger $\mathbf{J}$ Paed. 2012; 39(40):152-58.

6. AA. Beaven , BS. Atienza, VT. Abing,A. Phoebe,and T. Galleposo. Maternal constraints towards compliance to expanded program on immunization.Journal of Nursing and Health Care (JNHC). 2016; 3(2):30-38.

7. EW. Akoh, J Atudjieu, S.,Nouetchognou, MN. Yakun, FD Nemboi, SW. Sonkeng, SM. Fopa. The expanded program on immunization service delivery in Dschang health district west region of Cameroon .BioMed Central Public Health. 2016;16:1-8

8. WG. Cochrane. Sampling Techniques, 3rd Edition. New York: John Wiley and Son 1977.

9. A. Vikram, S. Amarjeet, S. Vijaylakshmi. Coverage and Quality of Immunization Services in Rural Chandigarh. Indian Pediatrics journal. 2012; 29:565 -567.

10. National Primary Health Care Development Agency. National routine immunization strategic plan: 2013-2015. Abuja, Nigeria: Federal Ministry of Health. 2013:10-12. 